\title{
HISTORIA DE LA FILOSOFÍA POLÍTICA: TRES TIPOS DE ANÁLISIS ${ }^{1}$
}

\author{
Luciano Venezia ${ }^{2}$ \\ Conicet / UNQ
}

Recibido: 21.12.2018 - Aceptado: 02.08.2019

\begin{abstract}
RESUMEN
En este ensayo distingo tres tipos de aproximación a los textos clásicos de la historia de la filosofía política, que llamo "reconstrucción", "ilustración” y "utilización”. Desarrollo brevemente cada una de las metodologías, basándome especialmente en mi propio trabajo sobre la obra de John Locke y Thomas Hobbes. Para finalizar, presento algunas ideas acerca del valor de los diferentes enfoques.

Palabras clave: Análisis filosófico; Historia de la filosofía política; John Locke; Thomas Hobbes.
\end{abstract}

\begin{abstract}
In this paper I outline three kinds of analysis of classical texts in the history of political philosophy, which I call "Reconstruction", "Illustration" and "Use". I briefly develop each of these methodologies, relying especially on my own work on John Locke and Thomas Hobbes. Finally, I put forward some thoughts about the value of the different kinds of approach.
\end{abstract}

Keywords: Philosophical Analysis; History of Political Philosophy; John Locke; Thomas Hobbes.

1 Agradezco los comentarios y sugerencias de Francisco García Gibson, Juan Ormeño Karzulovic, Plinio Junqueira Smith, Miguel Saralegui y Macarena Marey a versiones anteriores de este trabajo.

2 lvenezia@unq.edu.ar 


\section{INTRODUCCIÓN}

Derek Parfit acuñó una distinción sugestiva para caracterizar los dos tipos de análisis de las obras clásicas de la historia de la filosofía adoptados usualmente. Recientemente, Andrés Rosler articuló la distinción de Parfit en relación con las dos escuelas interpretativas de estudio de la historia del pensamiento político más difundidas en la literatura contemporánea. Por un lado, está el enfoque de los "arqueólogos", que puede ser asociado con la metodología de los historiadores agrupados en torno a la "Escuela de Cambridge". De acuerdo con esta perspectiva, los textos clásicos (incluyendo las obras centrales de la historia del pensamiento político) son fundamentalmente artefactos culturales situados históricamente. Por esta razón, los historiadores-arqueólogos consideran que estas obras deben ser analizadas en el marco de las convenciones lingüísticas que caracterizan al contexto ideológico del momento en que fueron escritas. Por otro lado, está la perspectiva de los "profanadores de tumbas", que en cambio puede relacionarse con la metodología adoptada por los filósofos políticos de Oxford; si se quiere, es el enfoque "analítico" o "sistemático" de estudio de los textos centrales de la historia de la filosofía y el pensamiento político. En este caso, los intérpretes-filósofos asumen que las obras clásicas son, ante todo, vehículos de ideas y argumentos más o menos trasparentes, lo que eventualmente permite que puedan utilizarse en el debate teórico contemporáneo (Rosler 2016, 13-16).

Por supuesto, la distinción entre una perspectiva históricoarqueológica y otra filosófico-analítica sugiere que se trata de dos tipos de enfoque fundamentalmente incompatibles. Sin embargo, como Rosler reconoce, los dos modos de abordaje pueden ser combinados, al menos hasta cierto punto $(2016,18-19) .^{3}$ Asimismo, la afirmación de que la metodología de los historiadores es "arqueológica" parece conllevar la idea de que esas interpretaciones, por sí solas, no tienen demasiado valor, al menos desde un punto de vista teórico o filosófico. De hecho, esta parece ser la posición de Rosler: "nos preocupamos por una obra [histórica] no solamente para comprender su significado, sino que además nos interesa

${ }^{3}$ Un ejemplo en este sentido son los textos de Kinch Hoekstra sobre Thomas Hobbes. Por supuesto, llevar a buen puerto una investigación que busca la combinación de los dos tipos de enfoques es una tarea extremadamente complicada. 
saber si tiene algo que agregar, si puede contribuir a la discusión contemporánea ofreciéndose como una alternativa a los paradigmas dominantes. [...] en última instancia, el atractivo o la valoración de un discurso, por político que sea, no puede depender de un contexto o de la historia" $(2016,19)$. Sin embargo, esta postura es demasiado estrecha. Por lo pronto, los historiadores de la filosofía obviamente consideran que su labor es teóricamente valiosa (Skinner 2004, 71-75; 2007, 218-222). Además, no parece haber nada objetable en desarrollar un análisis filosófico genuino de una obra que "no tiene nada que agregar". Por ejemplo, quizá podamos aprender cosas interesantes de teorías equivocadas.

De cualquier forma, la dicotomía planteada por Parfit y retomada por Rosler da cuenta de los dos tipos de análisis de los textos centrales de la historia de la filosofía política. Al mismo tiempo, la caracterización es demasiado general. Aun cuando seguramente es el enfoque dominante, hay muchos trabajos historiográficos que no siguen estrictamente la metodología cantabrigense. ${ }^{4}$ Por otro lado, no todas las lecturas analíticas son idénticas. En particular, en el presente trabajo muestro que hay tres modos de abordaje y discusión de los textos fundamentales de la historia de la filosofía política al interior de las interpretaciones de esta naturaleza que, aun cuando eventualmente pueden ser combinados, son fundamentalmente diferentes. Voy a llamar a estos tres tipos de análisis "reconstrucción", “ilustración" y "utilización". A fin de desarrollar las diferentes perspectivas, en lo que sigue me permito hacer referencia a mi propio trabajo de análisis de algunos textos centrales de la historia de la filosofía política moderna.

\section{RECONSTRUCCIÓN}

Las tesis y argumentos desarrollados en los textos clásicos de la historia de la filosofía política no son completamente transparentes; de hecho, algunas obras (por distintas razones) son especialmente difíciles, cuando no directamente oscuras. En este sentido, la primera consideración que lleva al análisis de una obra clásica es conocer exactamente el contenido sustantivo de la teoría desarrollada en el texto, lo que eventualmente nos va a permitir valorar críticamente sus diferentes tesis y argumentos. ${ }^{5}$

Ahora bien, los textos clásicos difícilmente desarrollan una "teoría política" o "filosofía política" en términos generales. En cambio, estas obras

4 De hecho, algunos críticos sostienen que Quentin Skinner, el representante más importante y relevante de la Escuela de Cambridge, en realidad no sigue esta metodología en sus trabajos sobre Hobbes. Ver: Collins 2009.

5 En cambio, el interés por el significado de una obra es fundamentalmente una preocupación de índole historiográfica. Ver: Rorty 1990, 75-76. 
incluyen consideraciones sobre problemas o cuestiones específicos. En otras palabras, una obra clásica, por más "sistemática" que sea, en definitiva trata sobre un tema o una serie de temas particulares; la teoría o filosofía política en cuestión involucra una posición sobre algo. ${ }^{6}$

Algunos de los temas tratados en una obra clásica pueden estar vigentes en la discusión teórica actual, mientras que otras cuestiones pueden estar completamente fuera de la agenda filosófica contemporánea. Por ejemplo, los textos canónicos de la tradición contractualista paradigmáticamente desarrollan argumentos destinados a justificar la obligación política de los ciudadanos, que es una problemática que está plenamente vigente en la filosofía política analítica contemporánea. Por esta razón, las interpretaciones analíticas de los textos centrales de la historia del pensamiento político se interesan fundamentalmente por un tópico específico; en general, se trata de una cuestión vigente en la filosofía política analítica actual, aun cuando ciertamente hay lecturas analíticas de temas de valor "histórico". Al mismo tiempo, la teoría en cuestión está a su vez articulada con consideraciones ulteriores, de manera tal que el análisis normalmente conlleva asimismo el estudio de las ideas relacionadas. Por ejemplo: los lectores analíticos del Leviatán seguramente se interesan en primera instancia por la teoría de la obligación política que Thomas Hobbes desarrolla en esta obra. A su vez, el análisis de la teoría de la obligación política de Hobbes conlleva naturalmente el estudio de los argumentos hobbesianos acerca del conflicto en el estado de naturaleza, en tanto ese escenario es el elemento que eventualmente permite que Hobbes arribe a las conclusiones sustantivas que constituyen su concepción particular de la obligación política. Finalmente, la discusión sobre el conflicto político tiene ramificaciones ulteriores. Dado que Hobbes considera que el elemento clave para dar cuenta de la autoridad del soberano y la obligación política de los súbditos es un acto de consentimiento personal, como el incluido en la formulación de una promesa o un contrato, las interpretaciones analíticas de su teoría de la obligación política consiguientemente normalmente incluyen el examen de la posición de Hobbes acerca de la naturaleza de la noción misma de obligación contractual.

De esta forma, la tarea incluida en una interpretación analítica medianamente atendible difícilmente consista en un mero resumen o exposición de las tesis principales desarrolladas en una obra clásica. Antes

6 Por supuesto, hay libros que se llaman "la filosofía política de Hobbes", "el pensamiento político de Locke", etc. Sin embargo, estos textos, en el mejor de los casos, tratan los varios temas que conforman la filosofía o teoría política del autor en cuestión. Al mismo tiempo, la mayoría de las veces, los libros de esta naturaleza son superficiales y no realizan aportes significativos. 
bien, la labor consiste fundamentalmente en la reconstrucción de los argumentos y tesis sustantivas que conforman una teoría filosófica articulada sobre un tópico particular.

Una característica distintiva de las interpretaciones analíticas es la utilización del aparato conceptual que forma parte de la filosofía moral y política analítica actual. Por ejemplo, en mi libro Hobbes on Legal Authority and Political Obligation utilizo el análisis del concepto de autoridad propuesto por Joseph Raz para desarrollar una reconstrucción analítica de la teoría de la autoridad y la obligación política de Hobbes. La idea clave de Raz es que las directivas de una autoridad no afectan el razonamiento práctico en virtud de su peso o fuerza relativa, sino que introducen razones para actuar de una clase o tipo particular. ${ }^{7}$ Los mandatos de una autoridad prevalecen sobre el resto de las consideraciones relevantes al momento de actuar; Raz destaca esta característica señalando que las directivas de una autoridad tienen un carácter "reemplazante" $(1986,46)$. Por supuesto, esta caracterización de la forma en que las órdenes de una autoridad afectan el razonamiento práctico es posterior a Hobbes. Al mismo tiempo, existen varios pasajes en los textos hobbesianos que permiten considerar que Hobbes analiza de manera similar la forma en que las directivas de la autoridad política afectan la deliberación de los súbditos. En concreto, la discusión de Hobbes del modo en que las prescripciones de un árbitro afectan la deliberación individual (2003, 67-68), así como su distinción entre consejos y mandatos $(2003,226-227)$, junto con la idea de que "es manifiesto que la ley, en general, no es consejo sino mandato" $(2003,233)$, ofrecen el basamento textual para desarrollar una interpretación raziana de su teoría de la autoridad y la obligación política (Venezia 2015, 41-62).

La reconstrucción articulada en el marco de una interpretación analítica es el primer paso de una tarea que normalmente incluye asimismo la evaluación crítica, sobre todo para defender la teoría en cuestión. Ahora bien, las obras clásicas muchas veces incluyen discusiones sobre cuestiones pasadas de moda; un ejemplo es el análisis de la mejor forma de gobierno. ${ }^{8}$ En este sentido, una lectura analítica puede tener por objeto la reconstrucción de una concepción errada o directamente falsa. Por ejemplo, difícilmente haya en la actualidad defensores de las posiciones sustantivas desa-

7 El mismo elemento caracteriza a nociones relacionadas, tales como decisiones, planes, promesas y ciertas normas o reglas.

8 Difícilmente encontremos hoy día una teoría filosófica defendiendo la monarquía o la aristocracia; el gobierno democrático está mayormente fuera de discusión en la filosofía política analítica actual. Al mismo tiempo, sí es una cuestión controvertida qué es lo que justifica la democracia. 
rrolladas por Hobbes; en efecto, pocos filósofos analíticos suscriben el principio de obligación política hobbesiano (Lloyd 1992, 51) que prescribe que "los súbditos deben a los soberanos simple obediencia en todas las cosas donde [actuar de esa manera] no sea incompatible con [la obediencia a] las leyes de Dios" $(2003,229)$. De esta manera, una reconstrucción analítica puede tener por objeto determinar qué está mal en una cierta concepción. En el contexto de mi interpretación de la teoría de la obligación política de Hobbes, el problema radica fundamentalmente en el alcance de los mandatos de la autoridad política. ${ }^{9}$

La utilización de teorías matemáticas modernas, sobre todo la teoría de juegos, constituye una práctica estándar en el marco de las interpretaciones analíticas de la filosofía política de Hobbes. Algunos trabajos hacen un uso relativamente accesible de estas herramientas, pero en algunos casos la discusión tiene un grado de sofisticación tal que la mera lectura de muchos de estos textos requiere de un entrenamiento específico. ${ }^{10}$ Por supuesto, los filósofos analíticos normalmente consideran que el uso de estos instrumentos está plenamente justificado. Por ejemplo, Jean Hampton escribe lo siguiente:

Hobbes quería que su obra fuera apreciada como un argumento filosófico a favor de la soberanía absoluta, no como una pieza de un museo de creencias políticas del siglo diecisiete. Por tanto, el uso de cualquier herramienta de lógica o cualquier distinción conceptual moderna que ayude a avanzar, clarificar o mejorar el argumento de Hobbes a favor de su teoría política, está en pleno acuerdo con sus propósitos y es compatible con el espíritu de su trabajo. $(1986,3)^{11}$

Dado que Hobbes obviamente no estaba al tanto de los desarrollos de la teoría de juegos, muchos críticos consideran que el uso de estas herramientas hace que las reconstrucciones analíticas sean necesariamente anacrónicas. Por ejemplo, Iain Hampsher-Monk suscribe esta tesis cuando afirma que "[l]a formalización de las insinuaciones de Hobbes ha generado un amplio tema [...] que supera de lejos cualquier cosa que se pudiera atribuir al propio Hobbes" $(1996,42)$. Esencialmente el mismo cuestionamiento puede hacerse respecto de la utilización de distinciones conceptuales o la imposición de ideas propias de la filosofía moral y política analítica actual.

A mi juicio, la afirmación de que el uso de análisis conceptuales modernos (como la caracterización raziana de la forma en que las directivas de una autoridad afectan la deliberación práctica) y de herramientas actuales (como la teoría de juegos) involucran una forma de anacronismo que no

\footnotetext{
${ }^{9}$ Para el análisis del alcance de una orden/razón autoritativa, ver: Raz 1991, 52.

10 Para un texto de esta naturaleza, ver: Vanderschraaf 2006.

11 Ver también: Hampton 1986, 137.
} 
tiene en cuenta cuál es el propósito de las interpretaciones analíticas. Por lo pronto, las reconstrucciones analíticas no pretenden realizar interpretaciones históricas; antes bien, estos trabajos buscan presentar teorías defendibles en el contexto del debate filosófico contemporáneo (Smith y Bolzani 2012, 363-364). En otras palabras, las interpretaciones analíticas no pretenden meramente describir lo que el autor de la obra escribió, sino en cambio articular la posición sustantiva particular que está desarrollada en sus textos (Wood 2002, 228). Por esta razón, una interpretación analítica puede incluir argumentos originales que, partiendo de las propias premisas del autor, permiten arribar a sus conclusiones. Asimismo, las interpretaciones analíticas pueden ofrecer consideraciones originales para decidir entre posiciones contradictorias sostenidas por un autor clásico a lo largo del tiempo o, incluso, en un mismo texto (Venezia 2013). Cuando tenemos en cuenta estas consideraciones, la crítica que señala que estas interpretaciones son anacrónicas pierde sentido.

\section{ILUSTRACIÓN}

Hay un segundo modo de analizar las obras clásicas de la historia del pensamiento político que, aunque está íntimamente conectado con la reconstrucción analítica, involucra en realidad un ejercicio diferente. Como señalé en la sección anterior, la reconstrucción normalmente tiene por objetivo desarrollar teorías defendibles en el contexto del debate filosófico-político contemporáneo. En cambio, esta forma alternativa de proceder apela a las obras clásicas de la historia del pensamiento político para ilustrar teorías susceptibles de ser desarrolladas y defendidas en la filosofía política analítica actual. En este sentido, la ilustración hace justicia a la tesis de que las obras políticas clásicas son vehículos de ideas y argumentos, lo que permite que puedan ser apropiadas en el marco del debate teórico contemporáneo.

De cualquier modo, la ilustración es relativamente infrecuente en la literatura reciente. Por mi parte, en mi libro La obligación política de los ciudadanos: una investigación filosófica, utilizo, en la medida de mis posibilidades, los textos clásicos de la historia del pensamiento político para ilustrar diferentes posiciones o tesis que pueden ser defendidas en el marco de la filosofía política analítica actual. En particular, en este texto muestro que las tesis y argumentos desarrollados en las obras de los escritores fundamentales de la filosofía política de los siglos XVII y XVIII (especialmente, los Dos ensayos sobre el gobierno civil y otros textos de Locke) son relevantes en el debate actual de la teoría de la obligación política. A continuación, voy a señalar la manera en que hago uso de los textos clásicos 
para ilustrar dos cuestiones diferentes: la discusión del contenido sustantivo del concepto de obligación política y la articulación de la teoría del consentimiento de la obligación política (Venezia 2019, capítulos 2 y 5).

En términos generales, hay dos análisis diferentes del contenido sustantivo de la noción de obligación política. De acuerdo con la primera versión, las obligaciones políticas de los ciudadanos consisten únicamente en la obligación de obedecer el derecho. Existe asimismo una caracterización alternativa que asegura que, además de la obligación de obediencia a las directivas de la autoridad política, la obligación política incluye deberes morales positivos que, aun cuando no estén legalmente prescritos, están típicamente asociados con el comportamiento de un "buen ciudadano". En particular, este segundo análisis considera que las obligaciones políticas de los ciudadanos incluyen la obligación de elegir autoridades públicas en elecciones democráticas (en jurisdicciones donde votar no es legalmente obligatorio) y la obligación de pelear en defensa del Estado cuando este lo reclama.

Las dos maneras de caracterizar el contenido sustantivo del concepto de obligación política están presentes en los textos centrales de la tradición contractualista. Este fenómeno permite ilustrar las dos posiciones sobre la base de ideas desarrolladas en algunas obras clásicas. Por un lado, Hobbes insiste en el Behemoth que la obligación de obedecer el derecho agota las obligaciones políticas de los ciudadanos:

La virtud de un súbdito está comprendida enteramente en la obediencia a las leyes de la república. Obedecer las leyes es justicia y equidad, que es la ley de la naturaleza, y por consiguiente es ley civil en todas las naciones del mundo; y nada es injusticia o iniquidad salvo lo que va contra la ley. $(2013,61)$

En cambio, Locke escribe en los Dos tratados sobre el gobierno civil que las obligaciones políticas de los ciudadanos incluyen la realización de aquellas acciones que son necesarias "para cumplir los fines para los que se han unido en sociedad" (1996, Segundo Ensayo, sección 99), lo que sugiere que, para Locke, el contenido sustantivo de la noción de obligación política es más amplio que la obligación de obedecer las directivas de la autoridad política.

Ahora voy a desarrollar las tesis centrales de la teoría del consentimiento de la obligación política. Por un lado, la teoría señala que actos de consentimiento personal, promesas o contratos son necesarios y normalmente suficientes para justificar las obligaciones políticas de los ciudadanos. Por otro lado, la teoría del consentimiento afirma que los ciudadanos efectivamente realizaron ciertas acciones voluntarias que 
conllevan la asunción de la obligación de obedecer a la autoridad política. Estas dos tesis, una de naturaleza conceptual y la otra de tipo empírico, están desarrolladas en los textos de Locke.

Locke articula la primera parte de la tesis conceptual en relación con su caracterización de la igualdad natural de los seres humanos. Por supuesto, el punto de partida de la concepción lockeana es la consideración de que las personas son naturalmente iguales (1996, Primer Ensayo, sección 51). Al mismo tiempo, Locke entiende la igualdad natural en términos fundamentalmente normativos; la igualdad natural consiste en "el igual derecho que tienen todos los hombres a su libertad natural, sin que nadie pueda verse sometido a la voluntad o autoridad de ningún otro" (1996, Segundo Ensayo, sección 54, énfasis en el original). De esta forma, la libertad natural tiene como resultado el hecho de que cada persona es soberana sobre sí misma; todas las personas son "reyes" por igual en el estado de naturaleza (1996, Segundo Ensayo, sección 123). Sobre la base de estas consideraciones, sostiene que el único modo en que las personas pueden adquirir obligaciones políticas es por medio de acciones que conllevan su consentimiento personal a la autoridad política: "[s]iendo los hombres libres e iguales e independientes por naturaleza, según hemos dicho ya, nadie puede salir de este estado y verse sometido al poder político de otro, a menos que medie su propio consentimiento" (1996, Segundo Ensayo, sección 95, énfasis en el original). ${ }^{12}$ De esta manera, Locke desarrolla la tesis de que los actos que conllevan el consentimiento personal son necesarios para justificar las obligaciones políticas de los ciudadanos.

Locke desarrolla asimismo la tesis que afirma que las promesas generan obligaciones morales. Según él, "[q]ue los hombres guarden sus compromisos es, sin duda, una importante e innegable regla moral" (1999, libro I, capítulo III, sección 5). ${ }^{13}$ Por esta razón, hasta "los forajidos y los ladrones, que han roto [vínculos comunes] con todo el resto del mundo, tienen que guardar la palabra y observar entre sí reglas de equidad, pues de lo contrario no podrían mantenerse unidos" (1999, libro I, capítulo III, sección 2). De hecho, considera que la fuerza normativa de este tipo de actos voluntarios es tan importante que obligan a Dios mismo. ${ }^{14}$ Locke consiguientemente afirma que "no hay nada más ridículo" que asumir voluntariamente una obligación y luego violarla. ${ }^{15}$ De acuerdo con él, "[t]oda comunidad entre seres humanos se derrumba" si las personas no cumplen

12 Ver también: Locke 1996, Primer Ensayo, sección 67; Segundo Ensayo, secciones $15,119,192$.

13 Ver también: Locke 1996, Segundo ensayo, sección 116.

${ }^{14}$ Locke 1996, Primer ensayo, sección 6; Segundo ensayo, sección 195.

${ }^{15}$ Locke 1996, Segundo ensayo, sección 194. Ver también: Locke 2011, 143. 
con sus obligaciones $(2007,13) .{ }^{16}$ Sobre la base de estas consideraciones, muestra que actos de consentimiento personal, como los que tienen lugar cuando una persona formula una promesa, son suficientes para generar obligaciones. ${ }^{17}$

La tesis empírica de la teoría del consentimiento de la obligación política recibe su articulación canónica en la teoría del consentimiento tácito lockeana, es decir, en la teoría que sostiene que los ciudadanos adquirieron la obligación de obedecer al Estado del que son miembros por medio de la realización de actos diferentes a la realización de acciones positivas cuyo significado convencional consiste en comunicar a otras personas la intención de asumir un conjunto de obligaciones particulares. Locke escribe que

Nadie niega que el consentimiento expreso de cualquier hombre para entrar en una sociedad lo convierte en un miembro de pleno derecho de esa sociedad, en súbdito de ese gobierno. La dificultad estriba en qué es lo que se considera un consentimiento tácito y hasta dónde llega el compromiso que se adquiere de esta manera, en el caso de que no lo haya expresado de ninguna manera. (1996, Segundo Ensayo, sección 119, énfasis en el original) ${ }^{18}$

A su vez, Locke sostiene que los ciudadanos consienten tácitamente a la autoridad del Estado al poseer o disfrutar de propiedad privada en un Estado e incluso por el solo hecho de residir dentro de los límites del Estado:

cualquier hombre que tenga alguna posesión o usufructo de alguna parte de los dominios de cualquier gobierno, da por ello su consentimiento tácito y está obligado a la obediencia de las leyes de ese gobierno como uno más, mientras dure el disfrute de esa propiedad. Y esto es así, tanto si se trata de una propiedad suya para siempre y para sus herederos, o si sólo está en ella por una semana, o si se trata de alguien que está viajando libremente por los caminos; y, en efecto, eso afecta a cualquiera que se encuentre dentro de los territorios de ese gobierno. (1996, Segundo Ensayo, sección 119, énfasis en el original) ${ }^{19}$

16 Ver también: Locke 2007, 14, 100.

17 Por supuesto, Locke reconoce que tienen que verificarse diferentes condiciones para que las promesas generen obligaciones. En primer lugar, Locke considera que la coerción invalida las obligaciones contractuales (1996, Segundo Ensayo, secciones 176, 186). Asimismo, Locke argumenta que las personas no pueden tener obligaciones contractuales con cualquier contenido. De hecho, Locke considera que no es posible tener obligaciones políticas para con un soberano absoluto sobre la base de que las personas no tienen el derecho a esclavizarse (1996, Segundo Ensayo, secciones 23, 24, 135, 137, 149, $168,171,172)$.

18 Ver también: Locke 2007, 52-53.

19 Ver también: Locke 1996, Segundo Ensayo, sección 73. Por cierto, la tesis de que la residencia continua es signo suficiente de consentimiento tácito a la autoridad del Estado es asimismo desarrollada por Jean-Jacques Rousseau, quien presenta argumentos similares en El contrato social (1975), libro IV, cap. II; ver también Platón, Critón (1966) 51d-52d. 


\section{UTILIZACIÓN}

Hay una tercera forma de proceder en el marco de una interpretación analítica de una obra clásica de la historia de la filosofía política. En este caso, la labor consiste en utilizar las ideas desarrolladas en un texto canónico para desarrollar una teoría pasible de ser defendida en el contexto de la filosofía política actual. A diferencia de la reconstrucción o de la ilustración, la utilización no tiene el propósito ofrecer una versión fiel de una obra o texto clásico. En realidad, la utilización normalmente conlleva la modificación de algunas tesis o ideas presentadas por el autor original.

Seguramente el ejemplo más destacado de utilización en la filosofía política analítica contemporánea es el argumento desarrollado por John Rawls, en Teoría de la justicia, a favor de su teoría de la "justicia como equidad". Rawls señala que

[l]o que he tratado de hacer es generalizar y llevar la teoría tradicional del contrato social representada por Locke, Rousseau y Kant, a un nivel más elevado de abstracción. [...] La teoría resultante es de naturaleza sumamente kantiana. De hecho no reclamo ninguna originalidad por las ideas que expongo. Las ideas fundamentales son clásicas y bien conocidas. Mi intención ha sido organizarlas dentro de un marco general usando ciertos recursos simplificadores con objeto de que la plenitud de su fuerza pueda ser apreciada. $(1995,9-10)$

Por supuesto, Rawls exagera; su teoría de la justicia como equidad es una contribución verdaderamente original. Al mismo tiempo, en Teoría de la justicia, Rawls hace un esfuerzo por mostrar que su concepción tiene particularmente un carácter kantiano. ${ }^{20}$ Por ejemplo Rawls desarrolla una "interpretación kantiana" de su teoría de la justicia distributiva en el parágrafo 40 del libro. Por otro lado, la idea de presentar esta teoría de la justicia distributiva como una concepción genuinamente "política", que Rawls formula en El liberalismo político (1996), conlleva precisamente mostrar que la concepción rawlsiana es susceptiblede ser caracterizada de manera independiente de las doctrinas comprehensivas que afirman los ciudadanos razonables, lo que implica abandonar el componente kantiano de la formulación original del argumento a favor de esta concepción.

Vale la pena detenerse asimismo en la utilización de las ideas de John Locke por parte de A. John Simmons. Simmons señala que la conjunción del voluntarismo político lockeano con la consideración de que los ciudadanos no realizaron los actos voluntarios necesarios para adquirir obligaciones políticas conlleva que los Estados son ilegítimos y consiguientemente que

20 Por cierto, varios especialistas en la filosofía política de Immanuel Kant sostienen que, en realidad, la teoría de Rawls no es verdaderamente kantiana. 
los ciudadanos no tenemos obligaciones políticas. De esta manera, Simmons acepta la tesis conceptual de la teoría de la obligación política de Locke descrita en la sección anterior y por consiguiente la posición desarrollada por este filósofo puede ser genuinamente caracterizada como "lockeana". Al mismo tiempo, Simmons rechaza la tesis empírica que forma parte de la teoría del consentimiento de Locke; según Simmons, David Hume y otros críticos de la teoría del consentimiento tienen razón cuando señalan que los ciudadanos no consintieron ni expresa ni tácitamente a la autoridad política (1979, capítulos III, IV y VIII; 1993, especialmente parte 4). ${ }^{21}$ Sobre la base de estas consideraciones, Simmons argumenta que la consecuencia natural del voluntarismo político lockeano es en realidad contraria a la defendida por el propio Locke. Simmons describe esta posición como "anarquismo filosófico a posteriori" (2001), de forma tal que contrasta esta teoría con las versiones conceptuales ("a priori") de anarquismo filosófico (Wolff 2004, especialmente capítulo 1).

De forma mucho más modesta, en mi libro sobre Hobbes discuto la teoría hobbesiana de los contratos realizados bajo coerción articulada por Hobbes y defendida en la actualidad por Claire Finkelstein. Por supuesto, una tesis central de la concepción desarrollada en el Leviatán es que las promesas y los contratos realizados bajo coerción introducen genuinas obligaciones morales. Hobbes desarrolla tres líneas de argumentación para defender esta tesis. En primer lugar, afirma que, de no ser así, los Estados existentes serían ilegítimos y consiguientemente los ciudadanos no tendrían obligaciones políticas. Hobbes cree que esta conclusión cuenta como una verdadera reductio ad absurdum, lo que le permite afirmar la tesis contraria: las promesas y los contratos realizados bajo coerción introducen verdaderas obligaciones morales. En segundo lugar, Hobbes sostiene que existen consideraciones de tipo consecuencialista para sostener esta idea; por ejemplo, los prisioneros de guerra serían, de otra manera, asesinados y consiguientemente no habría paz duradera entre los Estados. Finalmente, Hobbes articula la idea de que las promesas y los contratos realizados bajo coerción son moralmente vinculantes sobre la base de un análisis de la noción de voluntariedad. Las promesas y contratos realizados bajo coerción son realizados por miedo y, al menos en tanto ello no impida la deliberación, acciones de este tipo son genuinamente voluntarias y por consiguiente tienen consecuencias normativas $(2003,139-140)$.

${ }^{21}$ La crítica de Hume está desarrollada en "Del contrato original", en Ensayos políticos (1975). En este texto, asimismo, Hume desarrolla su propia posición acerca de la justificación de la obligación política. 
Finkelstein sostiene que hay dos consideraciones para admitir que el tercer argumento de Hobbes es válido:

\begin{abstract}
Hobbes estaba en lo correcto en rechazar la idea de que las acciones realizadas bajo coerción son involuntarias. En primer término, la persona que ejerce la coerción depende de la habilidad de su víctima de responder racionalmente a sus demandas para que sus esfuerzos en coercionarlo sean efectivos. Él depende de que su víctima realice un balanceo racional de males, y sería extraño decir que una acción es racional pero que sin embargo no es voluntaria. En segundo lugar, es difícil distinguir estos tipos de casos de aquellos en los cuales las opciones de uno son sencillamente poco atractivas. Si necesito desesperadamente comprar una pieza de pan y solo hay un tipo de pan en el mercado, normalmente no diríamos que compré ese tipo bajo coerción. Sería peor, aún, decir que lo compré involuntariamente. Ahora bien, ¿qué posible diferencia puede hacer el hecho de que en un caso una persona pone una pistola en mi cabeza, mientras que, en la otra, tengo razones imperiosas de un tipo diferente a favor de un curso de acción particular? (2001, 340-341, énfasis en el original)
\end{abstract}

A mi juicio, el razonamiento de Finkelstein es demasiado rápido; la racionalidad y la voluntariedad son dos nociones diferentes. En particular, hay acciones que son racionales, pero no son verdaderamente voluntarias, es decir, voluntarias en el sentido normativo requerido para poder decir que reflejan la agencia del que actúa de esa manera (Finkelstein 2001, 339). Apelando a un ejemplo altamente intuitivo (la manera en que Tom Hagen "convence" a Jack Woltz para que contrate al ahijado de Don Corleone para el rol protagónico de un film en El padrino), en mi libro sobre Hobbes argumento que, precisamente, las acciones realizadas bajo coerción (y por consiguiente las promesas y los contratos realizados bajo coerción) son voluntarias en el sentido psicológico de que los agentes toman una decisión racional sobre la base de una deliberación que tiene en cuenta las razones pro y contra para actuar de la forma requerida, pero que no son voluntarias en el sentido normativo requerido para poder afirmar que reflejan la agencia de los que son víctimas de la coerción. Antes bien, estas acciones reflejan la agencia de los que ejercen la coerción. Precisamente, a ello se debe que el uso de la coerción para obtener un fin sea normalmente inmoral. Como señala Friedrich A. Hayek, "[l]a coerción es mala precisamente porque [...] elimina al individuo como una persona pensante y valorizante y lo vuelve una mera herramienta para alcanzar los fines de otro" $(2011,71)$. Por esta razón, el análisis hobbesiano de Finkelstein, que afirma que las acciones realizadas bajo coerción son voluntarias, es a mi juicio errado; en cambio, la posición de sentido común acerca de esta cuestión es la correcta. Por tanto, la tesis hobbesiana de que las promesas y los contratos realizados bajo coerción introducen obligaciones moralmente vinculantes es falsa (Venezia 2015, 93-125). 


\section{CUÁL ES EL VALOR DEL ANÁLISIS DE LOS TEXTOS DE LA HISTORIA DE LA FILOSOFÍA POLÍTICA}

La cuestión que surge naturalmente luego de la identificación de los tres tipos de análisis de los textos centrales de la historia de la filosofía política es la consideración de su valor. Dado que la discusión de esta cuestión no es el objetivo principal de este trabajo, solamente voy a desarrollar algunas ideas en este sentido para finalizar.

Por lo pronto, considero que las obras centrales de la historia de la filosofía política son intrínsecamente valiosas. Por esta razón, creo que estudiar estos textos es asimismo una empresa valiosa. Al mismo tiempo, el valor de las interpretaciones analíticas es fundamentalmente instrumental. En definitiva, la reconstrucción, la ilustración y la utilización, cada una de manera diferente, involucran lecturas útiles de textos fundamentales de la cultura occidental.

Tal como señala Hampton en el pasaje citado más arriba, la principal utilidad de las reconstrucciones analíticas es que convierten las tesis y argumentos de los filósofos políticos del pasado en algo más que piezas de museo de creencias de otras épocas. Retomando la distinción de Parfit, no son únicamente objetos arqueológicos. En particular, las reconstrucciones analíticas permiten ver que las obras clásicas del pensamiento político constituyen verdaderas teorías filosóficas sobre cuestiones importantes; se trata de textos que, al ser reconstruidos analíticamente, incluyen argumentos sofisticados y distinciones conceptuales teóricamente relevantes.

La ilustración también es instrumentalmente valiosa, sobre todo desde un punto de vista didáctico. Por supuesto, muchas cuestiones que forman parte del debate filosófico-político actual son relativamente recientes. Con todo, al menos en algunos casos, las disputas del presente están relacionadas con discusiones teóricas desarrolladas en otras épocas de la historia del pensamiento político. Como mostré en el texto, la discusión contemporánea de la obligación política de los ciudadanos guarda una relación sumamente estrecha con el análisis de esta cuestión desarrollada en las obras centrales de la tradición contractualista de los siglos XVII y XVIII. En tanto utilizar textos de otras épocas para ilustrar debates actuales permite establecer relaciones de continuidad entre la historia del pensamiento político y la filosofía política analítica contemporánea, este modo de aproximación a las obras clásicas de la filosofía política es asimismo valioso.

El valor instrumental de la utilización de las obras centrales de la historia del pensamiento político es evidente. En definitiva, la utilización 
transforma a las obras del canon de la historia del pensamiento político en un insumo de la filosofía política analítica contemporánea. Por esta razón, el valor de la utilización de las obras de la historia de la filosofía política coincide en última instancia con el valor de la filosofía política. 


\section{BIBLIOGRAFIA}

Collins, Jeffrey. "Quentin Skinner's Hobbes and the Neo-Republican Project." Modern Intellectual History, vol. 6, № 2 (2009): 343-367.

Finkelstein, Claire. "A Puzzle about Hobbes on Self-Defense." Pacific Philosophical Quarterly, vol. 82, № 3-4 (2001): 332-361.

Hampton, Jean. Hobbes and the Social Contract Tradition. Cambridge: Cambridge University Press, 1986.

Hampsher-Monk, Iain. Historia del pensamiento político moderno. Los principales pensadores políticos de Hobbes a Marx, traducido por Ferran Meler. Barcelona: Crítica, 1996.

Hayek, Friedrich A. The Constitution of Liberty: The Definitive Edition, editado por Ronald Hamowy. Chicago y Londres: The University of Chicago Press, 2011.

Hobbes, Thomas. Leviatán, traducido por Antonio Escohotado. Buenos Aires: Losada, 2003.

—. Behemoth, traducido por Miguel Ángel Rodilla. Madrid: Tecnos, 2013.

Hume, David. "Del contrato original." En Ensayos políticos de David Hume, traducido por César Armando Gómez, 119-138. Madrid: Unión Editorial, 1975.

Lloyd, S. A. Ideals as Interests in Hobbes's Leviathan: The Power of Mind over Matter. Cambridge: Cambridge University Press, 1992.

Locke, John. Dos ensayos sobre el gobierno civil, traducido por Francisco Giménez Gracia. Barcelona: Planeta-De Agostini, 1996.

-. Ensayo sobre el entendimiento humano, traducido por Edmundo O’Gorman. México: Fondo de Cultura Económica, 1999.

—. "Moralidad." En "Ensayo sobre la tolerancia" y otros escritos sobre ética y obediencia civil de John Locke, traducido por Blanca Rodríguez López y Diego A. Fernández Peychaux, 141-144. Madrid: Biblioteca Nueva, 2011.

—. La Ley de la Naturaleza, traducido por Carlos Mellizo. Madrid: Tecnos, 2007.

Platón. Critón, traducido por Luis Noussan-Lettry. Buenos Aires: Editorial Universitaria de Buenos Aires, 1966.

Raz, Joseph. The Morality of Freedom. Oxford: Clarendon Press, 1986.

- Razón práctica y normas, traducido por Juan Ruiz Manero. Madrid: Centro de Estudios Constitucionales, 1991.

Rawls, John. Teoría de la justicia, traducido por María Dolores González. México: Fondo de Cultura Económica, 1995.

—. El liberalismo político, traducido por Antoni Domènech. Barcelona: Crítica, 1996.

Rosler, Andrés. Razones Públicas. Seis Conceptos Básicos sobre la República. Buenos Aires: Katz Editores, 2016. 
Rorty, Richard. "La historia de la filosofía: cuatro géneros." En La filosofía en la historia. Ensayos de historiografía de la filosofía, de Richard Rorty, J. B. Schneewind y Quentin Skinner, traducido por Eduardo Sinnott, 6999. Barcelona: Paidós, 1990.

Rousseau, Jean-Jacques. El contrato social, traducido por Fernando de los Ríos. Madrid: Espasa Calpe, 1975.

Simmons, A. John. Moral Principles and Political Obligations. Princeton: Princeton University Press, 1979.

-. On the Edge of Anarchy: Locke, Consent, and the Limits of Society. Princeton: Princeton University Press, 1993.

—. "Philosophical Anarchism." En Justification and Legitimacy: Essays on Rights and Obligations, de A. John Simmons, 102-121. Cambridge: Cambridge University Press, 2001.

Skinner, Quentin. La libertad antes del liberalismo, traducido por Fernando Escalante. México: Taurus, 2004.

—. "Interpretación y comprensión de los actos de habla." En Lenguaje, política e historia de Quentin Skinner, traducido por Cristina Fangmann, 185-222. Bernal: Universidad Nacional de Quilmes, 2007.

Smith, Plínio Junqueira, y Roberto Bolzani Filho. "Filosofía e historia de la filosofía." En Filosofía de la filosofía (Enciclopedia Iberoamericana de Filosofía 11) de Óscar Nudler, 349-372. Madrid: Trotta, 2012.

Vanderschraaf, Peter. "War or Peace? A Dynamical Analysis of Anarchy." Economics \& Philosophy, vol. 22, № 2 (2006): 243-279.

Venezia, Luciano. "Crucial Evidence: Hobbes on Contractual Obligation." Journal of the Philosophy of History, vol. 7, № 1 (2013): 106-135.

-. Hobbes on Legal Authority and Political Obligation. Basingstoke: Palgrave Macmillan, 2015.

—. La obligación política de los ciudadanos: una investigación filosófica, manuscrito sin publicar, 2019.

Wolff, Robert Paul. En defensa del anarquismo. Montevideo: Nordan-Comunidad, 2004.

Wood, Allen W. "What Dead Philosophers Mean." En Unsettling Obligations: Essays on Reason, Reality and the Ethics of Belief de Allen W. Wood, 213243. Stanford: CSLI Publications, 2002. 